\title{
Optimizing Daily Agent Scheduling in a Multiskill Call Center
}

\author{
Athanassios N. Avramidis \\ School of Mathematics, University of Southampton \\ Highfield, Southampton, SO17 1BJ, UNITED KINGDOM \\ Wyean Chan \\ Département d'informatique et de recherche opérationnelle \\ Université de Montréal, C.P. 6128, Succ. Centre-Ville \\ Montréal (Québec), H3C 3J7, CANADA \\ Michel Gendreau \\ Département d'informatique et de recherche opérationnelle and CIRRELT \\ Université de Montréal, C.P. 6128, Succ. Centre-Ville \\ Montréal (Québec), H3C 3J7, CANADA \\ Pierre L'Ecuyer \\ Département d'informatique et de recherche opérationnelle, CIRRELT and GERAD \\ Université de Montréal, C.P. 6128, Succ. Centre-Ville \\ Montréal (Québec), H3C 3J7, CANADA \\ Ornella Pisacane \\ Dipartimento di Elettronica Informatica e Sistemistica \\ Università della Calabria, Via P. Bucci, 41C \\ Arcavacata di Rende (CS), ITALY \\ January 19, 2009
}

\begin{abstract}
We examine and compare simulation-based algorithms for solving the agent scheduling problem in a multiskill call center. This problem consists in minimizing the total costs of agents under constraints on the expected service level per call type, per period, and aggregated. We propose a solution approach that combines simulation with integer or linear programming, with cut generation. In our numerical experiments with realistic problem instances, this approach performs better than all other
\end{abstract}


methods proposed previously for this problem. We also show that the two-step approach, which is the standard method for solving this problem, sometimes yield solutions that are highly suboptimal and inferior to those obtained by our proposed method.

\section{Introduction}

The telephone call center industry employs millions of people around the world and is fast growing. In the United States, for example, customer service representatives held 2.1 million jobs in 2004, and employment in this job category is expected to increase faster than average at least through 2014 (Bureau of Labor Statistics 2007). A few percent saving in workforce salaries easily means several million dollars.

Call centers often handle several types of calls distinguished by the required skills for delivering service. Training all agents to handle all call types is not cost-effective. Each agent has a selected number of skills and the agents are distinguished by the set of call types they can handle (also called their skill set). When such skill constraints exist, we speak of a multiskill call center. Skill-based routing (SBR), or simply routing, refers to the rules that control the call-to-agent and agent-to-call assignments. Most modern call centers perform skill-based routing (Koole and Mandelbaum 2002, Gans et al. 2003).

In a typical call center, inbound calls arrive at random according to some complicated stochastic processes, call durations are also random, waiting calls may abandon after a random patience time, some agents may fail to show up to work for any reason, and so on. Based on forecasts of call volumes, call center managers must decide (among other things) how many agents of each type (i.e., skill set) to have in the center at each time of the day, must construct working schedules for the available agents, and must decide on the call routing rules. These decisions are made under a high level of uncertainty. The goal is typically to provide the required quality of service at minimal cost.

The most common measure of quality of service is the service level (SL), defined as the longterm fraction of calls whose time in queue is no larger than a given threshold. Frequently, multiple measures of SL are of interest: for a given time period of the day, for a given call type, for a given combination of call type and period, aggregated over the whole day and all call types, and so on. For certain call centers that provide public services, SL constraints are imposed by external authorities, and violations may result in stiff penalties (CRTC 2000).

In this paper, we assume that we have a detailed stochastic model of the dynamics of the call center for one day of operation. This model specifies the stochastic processes for the call arrivals (these processes are usually non-stationary and doubly stochastic), the distributions of service times

and patience times for calls, the call routing rules, the periods of unavailability of agents between calls (e.g., to fill out forms, or to go to the restroom, etc.), and so forth. We formulate a stochastic optimization problem where the objective is to minimize the total cost of agents, under various SL 
constraints. This could be used in long-term planning, to decide how many agents to hire and for what skills to train them, or for short-term planning, to decide which agents to call for work on a given day and what would be their work schedule. The problem is difficult because for any given fixed staffing of agents (the staffing determines how many agents of each type are available in each time period), no reliable formulas or quick numerical algorithms are available to estimate the SL; it can be estimated accurately only by long (stochastic) simulations. Scheduling problems are in general NP-hard, even in deterministic settings where each solution can be evaluated quickly and exactly. When this evaluation requires costly and noisy simulations, as is the case here, solving the problem exactly is even more difficult and we must settle with methods that are partly heuristic.

Staffing in the single-skill case (i.e., single call type and single agent type) has received much attention in the call center literature. Typically, the workload varies considerably during the day (Gans et al. 2003, Avramidis et al. 2004, Brown et al. 2005), and the planned staffing can change only at a few discrete points in time (e.g., at the half hours). It is common to divide the day into several periods during which the staffing is held constant and the arrival rate does not vary much. If the system can be assumed to reach steady-state quickly (relative to the length of the periods), then steady-state queueing models are likely to provide a reasonably good staffing recommendation for each period. For instance, in the presence of abandonments, one can use an Erlang-A formula to determine the minimal number of agents for the required SL in each period (Gans et al. 2003). When that number is large, it is often approximated by the square root safety staffing formula, based on the Halfin-Whitt heavy-traffic regime, and which says roughly that the capacity of the system should be equal to the workload plus some safety staffing which is proportional to the square root of the workload (Halfin and Whitt 1981, Gans et al. 2003). This commonly used heuristic, known as the stationary independent period by period (SIPP) approach, often fails to meet target SL because it neglects the non-stationarity (Green et al. 2003). Non-stationary versions of these approximations have also been developed, still for the single-skill case (Jennings et al. 1996, Green et al. 2003).

Scheduling problems are often solved in two separate steps (Mehrotra 1997): After an appropriate staffing has been determined for each period in the first step, a minimum-cost set of shifts that covers this staffing requirement can be computed in the second step by solving a linear integer program. However, the constraints on admissible working shifts often force the second step solution to overstaff in some of the periods. This drawback of the two-step approach has been pointed out by several authors, who also proposed alternatives (Keith 1979, Thompson 1997, Henderson and Mason 1998, Ingolfsson et al. 2003, Atlason et al. 2004). For example, the SL constraint is often only for the time-aggregated (average) SL over the entire day; in that case, one may often obtain a lower-cost scheduling solution by reducing the minimal staffing in one period and increasing it in another period. Atlason et al. (2004) developed a simulation-based methodology to optimize agents' scheduling in the presence of uncertainty and general SL constraints, based on simulation and cutting-plane ideas. Linear inequalities (cuts) are added to an integer program until its optimal 
solution satisfies the required SL constraints. The SL and the cuts are estimated by simulation.

In the multiskill case, the staffing and scheduling problems are more challenging, because the workload can be covered by several possible combinations of skill sets, and the routing rules also have a strong impact on the performance. Staffing a single period in steady-state is already difficult; the Erlang formulas and their approximations (for the SL) no longer apply. Simulation seems to be the only reliable tool to estimate the SL. Cez̧ik and L'Ecuyer adapt the simulation-based methodology of Atlason et al. (2004) to the optimal staffing of a multiskill call center for a single period. They point out difficulties that arise with this methodology and develop heuristics to handle them. Avramidis et al. (2006) solve the same problem by using neighborhood search methods combined with an analytical approximation of SLs, with local improvement via simulation at the end. Pot et al. (2007) impose a constraint only on the aggregate SL (across all call types); they solve Lagrangean relaxations using search methods and analytical approximations.

Some authors have studied the special case where there are only two call types, and some have developed queueing approximations for the case of two call types, via Markov chains and under simplifying assumptions; see Stolletz and Helber (2004) for example. But here we are thinking of 20 to 50 call types or more, which is common in modern call centers, and for which computation via these types of Markov chain models is clearly impractical.

For the multiskill scheduling problem, Bhulai et al. (2007) propose a two-step approach in which the first step determines a staffing of each agent type for each period, and the second step computes a schedule by solving an IP in which this staffing is the right-hand side of key constraints. A key feature of the IP model is that the staff-coverage constraints allow downgrading an agent into any alternative agent type with smaller skill set, temporarily and separately for each period. Bhulai et al. (2007) recognize that their two-step approach is generally suboptimal and they illustrate this by examples.

In this paper, we propose a simulation-based algorithm for solving the multiskill scheduling problem, and compare it to the approach of Bhulai et al. (2007). This algorithm extends the method of Cez̧ik and L'Ecuyer, which solves a single-period staffing problem. In contrast to the two-step approach, our method optimizes the staffing and the scheduling simultaneously. Our numerical experiments show that our algorithm provides approximate solutions to large-scale realistic problem instances in reasonable time (a few hours). These solutions are typically better, sometimes by a large margin (depending on the problem), than the best solutions from the two-step approach. We are aware of no competitive faster method.

The remainder of this paper is organized as follows. In section 2, we formally define the problem at hand and provide a mathematical programming formulation. The new algorithm is described in 3. We report computational results on several test instances in section 4. The conclusion follows. A preliminary version of this paper was presented at the 2007 Industrial Simulation Conference (Avramidis et al. 2007a). 


\section{Model Formulation}

We now provide definitions of the multiskill staffing and scheduling problems. We assume that we have a stochastic model of the call center, under which the mathematical expectations used below are well defined, and that we can simulate the dynamics of the center under this model. Our problem formulations here do not depend on the details of this model.

There are $K$ call types, labeled from 1 to $K$, and $I$ agent types, labeled from 1 to $I$. Agent type $i$ has the skill set $S_{i} \subseteq\{1, \ldots, K\}$. The day is divided into $P$ periods of given length, labeled from 1 to $P$. The staffing vector is $\mathbf{y}=\left(y_{1,1}, \ldots, y_{1, P}, \ldots, y_{I, 1}, \ldots, y_{I, P}\right)^{\mathrm{t}}$ where $y_{i, p}$ is the number of agents of type $i$ available in period $p$. Given $\mathbf{y}$, the service level (SL) in period $p$ for type- $k$ calls is defined as

$$
g_{k, p}(\mathbf{y})=\mathbb{E}\left[C_{\mathrm{g}, k, p}\right] / \mathbb{E}\left[C_{k, p}+A_{k, p}\right]
$$

where $\mathbb{E}$ denotes the mathematical expectation, $C_{k, p}$ is the number of type- $k$ calls that arrive in period $p$ and eventually get served, $C_{\mathrm{g}, k, p}$ is the number of those calls that get served after waiting at most $\tau_{k, p}$ (a constant called the acceptable waiting time), and $A_{k, p}$ is the number of those calls that abandon after waiting at least $\tau_{k, p}$. Aggregate SLs, per call type, per period, and globally, are defined analogously. Given acceptable waiting times $\tau_{p}, \tau_{k}$, and $\tau$, the aggregate SLs are denoted by $g_{p}(\mathbf{y}), g_{k}(\mathbf{y})$ and $g(\mathbf{y})$ for period $p$, call type $k$, and overall, respectively.

A shift is a time pattern that specifies the periods in which an agent is available to handle calls. In practice, it is characterized by its start period (the period in which the agent starts working), break periods (the periods when the agent stops working), and end period (the period when the agent finishes his/her workday). In general, agents have several breaks of different duration; for instance, morning and afternoon coffee breaks, as well as a longer lunch break.

Let $\{1, \ldots, Q\}$ be the set of all admissible shifts. To simplify the exposition, we assume that this set is the same for all agent types; this assumption could easily be relaxed if needed, by introducing specific shift sets for each agent type. The admissible shifts are specified via a $P \times Q$ matrix $\mathbf{A}_{0}$ whose element $(p, q)$ is $a_{p, q}=1$ if an agent with shift $q$ works in period $p$, and 0 otherwise. A vector $\mathbf{x}=\left(x_{1,1}, \ldots, x_{1, Q}, \ldots, x_{I, 1}, \ldots, x_{I, Q}\right)^{\mathrm{t}}$, where $x_{i, q}$ is the number of agents of type $i$ working shift $q$, is a schedule. The cost vector is $\mathbf{c}=\left(c_{1,1}, \ldots, c_{1, Q}, \ldots, c_{I, 1}, \ldots, c_{I, Q}\right)^{\mathrm{t}}$, where $c_{i, q}$ is the cost of an agent of type $i$ with shift $q$. To any given shift vector $\mathbf{x}$, there corresponds the staffing vector $\mathbf{y}=\mathbf{A x}$, where $\mathbf{A}$ is a block-diagonal matrix with $I$ identical blocks $\mathbf{A}_{0}$, if we assume that each agent of type $i$ works as a type- $i$ agent for his/her entire shift.

However, following Bhulai et al. (2007), we also allow an agent of type $i$ to be temporarily downgraded to an agent with smaller skill set, i.e., of type $i_{p}$ where $S_{i_{p}} \subset S_{i}$, in any time period $p$ of his/her shift. Define $\mathscr{S}_{i}^{+}=\left\{j: S_{j} \supset S_{i} \wedge \nexists m: S_{j} \supset S_{m} \supset S_{i}\right\}\left(\mathscr{S}_{i}^{+}\right.$is thus the set of agent types whose skill set is a minimum strict superset of the skill set of agent type $i$ ) and $\mathscr{S}_{i}^{-}=\left\{j: S_{j} \subset S_{i} \wedge /\right.$ $\left.\exists m: S_{j} \subset S_{m} \subset S_{i}\right\}\left(\mathscr{S}_{i}^{-}\right.$is thus the set of agent types whose skill set is a maximum strict subset of skill set of agent type $i$ ). To illustrate, consider a call centre with $K=3$ call types, $I=4$ agent 
types, and skill sets $S_{1}=\{1\}, S_{2}=\{2\}$ (specialist agents), $S_{3}=\{2,3\}$, and $S_{4}=\{1,2,3\}$ (generalist agents); then we have, among others, $\mathscr{S}_{1}^{-}=\mathscr{S}_{2}^{-}=\emptyset, \mathscr{S}_{2}^{+}=\{3\}$, and $\mathscr{S}_{4}^{-}=\{1,3\}$. For each $i$ and $j \in \mathscr{S}_{i}^{-}$and each period $p$, we define the skill transfer variable $z_{i, j, p}$, which represents the number of type- $i$ agents that are temporarily downgraded to type $j$ during period $p$. Note that by performing multiple skill transfers during a given period, an agent of type $i$ may end up being downgraded to any type whose skill set included in $S_{i}$ (in the previous example, a type 4 agent could be downgraded to type 3 and then to type 2, even though there are no $z_{4,2, p}$ variables).

A schedule $\mathbf{x}=\left(x_{1,1}, \ldots, x_{1, Q}, \ldots, x_{I, 1}, \ldots, x_{I, Q}\right)^{\mathrm{t}}$ is said to cover the staffing $\mathbf{y}=\left(y_{1,1}, \ldots, y_{1, P}, \ldots\right.$, $\left.y_{I, 1}, \ldots, y_{I, P}\right)^{\mathrm{t}}$ if for $i=1, \ldots, I$ and $p=1, \ldots, P$, there are nonnegative integers $z_{j, i, p}$ for $j \in \mathscr{S}_{i}^{+}$and $z_{i, j, p}$ for $j \in \mathscr{S}_{i}^{-}$, such that

$$
\sum_{q=1}^{Q} a_{p, q} x_{i, q}+\sum_{j \in \mathscr{S}_{i}^{+}} z_{j, i, p}-\sum_{j \in \mathscr{S}_{i}^{-}} z_{i, j, p} \geq y_{i, p}
$$

These inequalities can be written in matrix form as $\mathbf{A x}+\mathbf{B z} \geq \mathbf{y}$, where $\mathbf{z}$ is a column vector whose elements are the $z_{i, j, p}$ variables and $\mathbf{B}$ is a matrix whose entries are in the set $\{-1,0,1\}$. With this notation, the scheduling problem can be formulated as

(P0) : [Scheduling problem]

$$
\begin{aligned}
\min & \mathbf{c}^{\mathrm{t}} \mathbf{x}=\sum_{i=1}^{I} \sum_{q=1}^{Q} c_{i, q} x_{i, q} \\
\text { s.t. } & \\
& \mathbf{A x}+\mathbf{B z} \geq \mathbf{y} \\
& g_{k, p}(\mathbf{y}) \geq l_{k, p} \text { for } 1 \leq k \leq K \text { and } 1 \leq p \leq P \\
& g_{p}(\mathbf{y}) \geq l_{p} \text { for } 1 \leq p \leq P \\
& g_{k}(\mathbf{y}) \geq l_{k} \text { for } 1 \leq k \leq K \\
& g(\mathbf{y}) \geq l \\
& \mathbf{x} \geq 0, \mathbf{z} \geq 0, \mathbf{y} \geq 0 \text { and integer }
\end{aligned}
$$

where $l_{k, p}, l_{p}, l_{k}$ and $l$ are given constants.

In practice, a given agent often works more efficiently (faster) when handling a smaller number of call types (i.e., if his/her skill set is artificially reduced). The possibility of downgrading agents to a smaller skill set for some periods can sometimes be exploited to take advantage of this increased efficiency. In case where the agent's speed for a given call type (in the model) does not depend on his/her skill set, one might think intuitively that downgrading cannot help, because it only limits the flexibility of the routing. This would be true if we had an optimal dynamic routing of calls. But in practice, an optimal dynamic routing is too complicated to compute and simpler routing rules are used instead. These simple rules are often static. Then, downgrading may sometimes help by effectively changing the routing rules. Clearly, the presence of skill transfer variables in (P0) cannot increase the optimal cost, it can only reduce it. 
Suppose we consider a single period, say period $p$, and we replace $g_{k, p}(\mathbf{y})$ and $g_{p}(\mathbf{y})$ by approximations that depend on the staffing of period $p$ only, say $\tilde{g}_{k, p}\left(y_{1, p}, \ldots, y_{I, p}\right)$ and $\tilde{g}_{p}\left(y_{1, p}, \ldots, y_{I, p}\right)$, respectively. If all system parameters are assumed constant over period $p$, then natural approximations are obtained by assuming that the system is in steady-state over this period. The single-period multiskill staffing problems can then be written as

$$
\begin{aligned}
\text { (P1) : } & {[\text { Staffing problem }] } \\
\min & \sum_{i=1}^{I} c_{i} y_{i} \\
\text { s.t. } & \\
& \tilde{g}_{k}\left(y_{1}, \ldots, y_{I}\right) \geq l_{k} \quad \text { for } 1 \leq k \leq K \\
& \tilde{g}\left(y_{1}, \ldots, y_{I}\right) \geq l \\
& y_{i} \geq 0 \quad \text { and integer for all } i
\end{aligned}
$$

where $c_{i}$ is the cost of agent type $i$ (for a single period), and the period index was dropped throughout. Simulation-based solution methods for this problem are proposed in Cez̧ik and L'Ecuyer and Avramidis et al. (2006). Pot et al. (2007) address a restricted version of it, with a single constraint on the aggregate SL over the period (i.e., they assume $l_{k}=0$ for all $k$ ).

In the approach of Bhulai et al. (2007), the first step is to determine an appropriate staffing, $\hat{\mathbf{y}}=\left(\hat{y}_{1,1}, \ldots, \hat{y}_{1, P}, \ldots, \hat{y}_{I, 1}, \ldots, \hat{y}_{I, P}\right)^{\mathrm{t}}$. For this, they look at each period $p$ in isolation and solve a version of (P1) with a single constraint on the aggregate SL; this gives $\hat{y}_{1, p}, \ldots, \hat{y}_{I, p}$ for each $p$. In their second step, they find a schedule that covers this staffing by solving:

\begin{tabular}{|rl|}
\hline (P2) : & [Two-stage approach] \\
$\min$ & $\mathbf{c}^{\mathrm{t}} \mathbf{x}$ \\
s.t. & \\
& $\mathbf{A x}+\mathbf{B z} \geq \hat{\mathbf{y}} \quad$ \\
& $\mathbf{x} \geq 0, \mathbf{z} \geq 0 \quad$ and integer
\end{tabular}

The presence of skill-transfer variables generally reduces the optimal cost in (P2) by adding flexibility, compared with the case where no downgrading is allowed. However, there sometimes remains a significant gap between the optimal solution of (P0) and the best solution found for the same problem by the two-step approach. The following simplified example illustrates this.

Example 1 Let $K=I=P=3$, and $Q=1$. The single type of shift covers the three periods. The skill sets are $S_{1}=\{1,2\}, S_{2}=\{1,3\}$, and $S_{3}=\{2,3\}$. All agents have the same shift and the same cost. Suppose that the total arrival process is stationary Poisson with mean 100 per minute. This incoming load is equally distributed between call types $\{1,2\}$ in period $1,\{1,3\}$ in period $2,\{2,3\}$ 
in period 3. Any agent can be downgraded to a specialist that can handle a single call type (that belongs to his skill set), in any period. In the presence of such specialists, an incoming call goes first to its corresponding specialist if there is one available, otherwise it goes to a generalist that can handle another call type as well. When the agent becomes available, he serves the call that has waited the longest among those in the queue (if any). The service times are exponential with mean 1 per minute, there are no abandonments, and the SL constraints specify that $80 \%$ of all calls must be served within 20 seconds, in each time period, on average over an infinite number of days.

If we assume that the system operates in steady-state in period 1, then the optimal staffing for that period is 104 agents of type 1 . Since all agents can serve all calls, we have in this case an $M / M / s$ queue with $s=104$, and the global SL is $83.4 \%$, as can be computed by the well-known Erlang-C formula (Gans et al. 2003). By symmetry, the optimal staffing solutions for the other periods are obviously the same: 104 agents of type 2 in period 2 and 104 agents of type 3 in period 3 . Then, the two-step approach gives a solution to (P2) with 104 agents of each type, for a total of 312 agents.

Solving (P0) directly instead (e.g., using the simulation-based algorithm described in the next section), assuming again (as an approximation) that the system is in steady-state in each of the three periods, we find a feasible solution with 35 agents of type 1, 35 agents of type 2, and 34 agents of type 3 , for a total of 104 agents. With this solution, during period 1, the agents of types 2 and 3 are downgraded to specialists who handle only call types 1 and 2, respectively, and the agents of type 1 act as generalists. A similar arrangement applies to the other periods, mutatis mutandis. Note that this solution of (P0) remains valid even if we remove the skill transfer variables from the formulation of (P0), because the sets $S_{i}^{-}$and $S_{i}^{+}$are all empty, if we assume that the routing rules do not change; i.e., if calls are always routed first to agents that can handle only this call type among the calls that can arrive during the current period.

Suppose now that we add the additional skill sets $S_{4}=\{1\}, S_{5}=\{2\}, S_{6}=\{3\}$, and that these new specialists cost 6 each, whereas the agents with two skills cost 7 . In this case it becomes attractive to use specialists to handle a large fraction of the load, because they are less expensive, and to keep a few generalists in each period to obtain a "resource sharing" effect. It turns out that an optimal staffing solution for period 1 is 2 generalists (type 1) and 52 specialists of each of the types 4 and 5. An analogous solution holds for each period. With these numbers, if downgrading is not possible, the two-step approach gives a solution with 6 generalists ( 2 of each type) and 156 specialists (52 of each type), for a total cost of 978. If downgrading is allowed, then the two-step approach finds the following much better solution: 2 agents of type 1 and 52 of each of the types 2 and 3, for a total cost of 742. The skill transfer works in this way. In period 1: 52 agents of type 2 are downgraded to specialists of type 4 and 52 of type 3 to specialists of type 5 . In period 2: 2 agents of type 1 are downgraded to agents of type 4, 52 of type 2 to type 6 and 50 of type 3 to type 4. In period 3: 2 agents of type 1 are downgraded to agents of type 5, 50 of type 2 to type 5 and 52 of type 3 to type 6 . If we solve (P0) directly with these additional skill sets, we get the same solution 
as without them; i.e., 104 agents with two skills each, for a total cost of 728 . This is again better than with the two-step approach, but the gap is much smaller than what we had with only three skill sets.

Example 2 In the previous example, if all the load was from a single call type, there would be a single agent type and the two-step approach would provide exactly the same solution as the optimal solution of (P0). The example illustrates a suboptimality gap due to a variation in the type of load.

Another potential source of suboptimality (this one can occur even in the case of a single call type) is the time variation of the total load from period to period. If there is only a global SL constraint over the entire day, then the optimal solution may allow a lower SL during one (or more) peak period(s) and recover an acceptable global SL by catching up in the other periods. To account for this, Bhulai et al. (2007), Section 5.4, propose a heuristic based on the solution obtained by their basic two-step approach. Although this appears to work well in their examples, the effectiveness of this heuristic for general problems is not clear.

Yet another type of limitation that can significantly increase the total cost is the restriction on the set of available shifts. Suppose for example that there is a single call type, that the day has 10 periods, and that all shifts must cover 8 periods, with 7 periods of work and a single period of lunch break after 3 or 4 periods of work. Thus a shift can start in period 1, 2, or 3, and there are six shift types in total. Suppose we need 100 agents available in each period. For this we clearly need 200 agents, each one working for 7 periods, for a total of 1400 agent-periods. If there were no constraints on the duration and shape of shifts, on the other hand, then 1000 agent-periods would suffice.

\section{Optimization by Simulation and Cutting Planes}

We now describe the proposed simulation-based optimization algorithm. The general idea is to replace the problem $(\mathrm{P} 0)$ by a sample version of it, $\left(\mathrm{SP}_{n}\right)$, and then replace the nonlinear SL constraints by a small set of linear constraints, in a way that the optimal solution of the resulting relaxed sample problem is close to that of (P0). The relaxed sample problem is solved by linear or integer programming.

We first describe how the relaxation works when applied directly to (P0); it works the same way when applied to the sample problem. Consider a version of (P0) in which the SL constraints have been replaced by a small set of linear constraints that do not cut out the optimal solution. Let $\overline{\mathbf{y}}$ be the optimal solution of this (current) relaxed problem. If $\overline{\mathbf{y}}$ satisfies all SL constraints of (P0), then it is an optimal solution of (P0) and we are done. Otherwise, take a violated constraint of (P0), say $g(\overline{\mathbf{y}})<l$, suppose that $g$ is (jointly) concave in $\mathbf{y}$ for $\mathbf{y} \geq \overline{\mathbf{y}}$, and that $\overline{\mathbf{q}}$ is a subgradient of $g$ at $\overline{\mathbf{y}}$. Then

$$
g(\mathbf{y}) \leq g(\overline{\mathbf{y}})+\overline{\mathbf{q}}^{\mathrm{t}}(\mathbf{y}-\overline{\mathbf{y}})
$$


for all $\mathbf{y} \geq \overline{\mathbf{y}}$. We want $g(\mathbf{y}) \geq l$, so we must have

$$
l \leq g(\mathbf{y}) \leq g(\overline{\mathbf{y}})+\overline{\mathbf{q}}^{\mathrm{t}}(\mathbf{y}-\overline{\mathbf{y}})
$$

i.e.,

$$
\overline{\mathbf{q}}^{\mathrm{t}} \mathbf{y} \geq \overline{\mathbf{q}}^{\mathrm{t}} \overline{\mathbf{y}}+l-g(\overline{\mathbf{y}}) .
$$

Adding this linear cut inequality to the constraints removes $\overline{\mathbf{y}}$ from the current set of feasible solutions of the relaxed problem without removing any feasible solution of (P0). On the other hand, in case $\overline{\mathbf{q}}$ is not really a subgradient (which may happens in practice), then we may cut out feasible solutions of (P0), including the optimal one. We will return to this.

Since we cannot evaluate the functions $g$ exactly, we replace them by a sample average over $n$ independent days, obtained by simulation. Let $\omega$ represent the sequence of independent uniform random numbers that drives the simulation for those $n$ days. When simulating the call center for different values of $\mathbf{y}$, we assume that the same uniform random numbers are used for the same purpose for all values of $\mathbf{y}$, for each day. That is, we use the same $\omega$ for all $\mathbf{y}$. Proper synchronization of these common random numbers is implemented by using a random number package with multiple streams and substreams (Law and Kelton 2000, L'Ecuyer et al. 2002, L'Ecuyer 2004).

The empirical $S L$ over these $n$ simulated days is a function of the staffing $\mathbf{y}$ and of $\omega$. We denote it by $\hat{g}_{n, k, p}(\mathbf{y}, \omega)$ for call type $k$ in period $p ; \hat{g}_{n, p}(\mathbf{y}, \omega)$ aggregated over period $p ; \hat{g}_{n, k}(\mathbf{y}, \omega)$ aggregated for call type $k$; and $\hat{g}_{n}(\mathbf{y}, \omega)$ aggregated overall. For a fixed $\omega$, these are all deterministic functions of $\mathbf{y}$. Instead of solving directly ( $\mathrm{P} 0)$, we solve its sample-average approximation $\left(\mathrm{SP} 0_{n}\right)$ obtained by replacing the functions $g$ in (P0) by their sample counterparts $\hat{g}$ (here, $\hat{g}$ stands for any of the empirical SL functions, and similarly for $g$ ).

We know that $\hat{g}_{n, k, p}(\mathbf{y})$ converges to $g_{k, p}(\mathbf{y})$ with probability 1 for each $(k, p)$ and each $\mathbf{y}$ when $n \rightarrow \infty$. In this sense, $\left(\mathrm{SP} 0_{n}\right)$ converges to $(\mathrm{P} 0)$ when $n \rightarrow \infty$. Suppose that we eliminate a priori all but a finite number of solutions for (P0). This can easily be achieved by eliminating all solutions for which the total number of agents is unreasonably large. Let $\mathscr{Y}^{*}$ be the set of optimal solutions of (P0) and suppose that no SL constraint is satisfied exactly for these solutions. Let $\mathscr{Y}_{n}^{*}$ be the set of optimal solutions of $\left(\mathrm{SP}_{n}\right)$. Then, the following theorem implies that for $n$ large enough, an optimal solution to the sample problem is also optimal for the original problem. It can be proved by a direct adaptation of the results of Vogel (1994) and Atlason et al. (2004); see also Cez̧ik and L'Ecuyer.

Theorem 1 With probability 1 , there is an integer $N_{0}<\infty$ such that for all $n \geq N_{0}, \mathscr{Y}_{n}^{*}=\mathscr{Y}^{*}$. Moreover, suppose that the service-level estimators satisfy the standard large-deviation principle ( $a$ mild assumption): For every $\varepsilon>0$, there are positive integers $N_{0}$ and $\kappa$ such that for all $n \geq N_{0}$ and $\mathbf{y} \in \mathscr{Y}, P\left(\left|\hat{g}_{n, k, p}(\mathbf{y}, \omega)-g_{k, p}(\mathbf{y})\right|>\varepsilon\right) \leq e^{-n \kappa}$ for all $k, p$, and for the aggregate service-levels as well. Then, there are positive real numbers $\alpha$ and $\beta$ such that for all $n$,

$$
P\left[\mathscr{Y}_{n}^{*}=\mathscr{Y}^{*}\right] \geq 1-\alpha e^{-\beta n} .
$$


We solve $\left(\mathrm{SPO}_{n}\right)$ by the cutting plane method described earlier, with the functions $g$ replaced by their empirical counterparts. The major practical difficulty is to obtain the subgradients $\overline{\mathbf{q}}$. In fact, the functions $\hat{g}$ in the empirical problem (computed by simulation) are not necessarily concave for finite $n$, even in the areas where the functions $g$ of (P0) are concave. To obtain a (tentative) subgradient $\overline{\mathbf{q}}$ of a function $\hat{g}$ at $\overline{\mathbf{y}}$, we use forward finite differences as follows. For $j=1, \ldots, I P$, we choose an integer $d_{j} \geq 0$, we compute the function $\hat{g}$ at $\overline{\mathbf{y}}$ and at $\overline{\mathbf{y}}+d_{j} \mathbf{e}_{j}$ for $j=1, \ldots, I P$, where $\mathbf{e}_{j}$ is the $j$ th unit vector, and we define $\overline{\mathbf{q}}$ as the $I P$-dimensional vector whose $j$ th component is

$$
\bar{q}_{j}=\left[\hat{g}\left(\overline{\mathbf{y}}+d_{j} \mathbf{e}_{j}\right)-\hat{g}(\overline{\mathbf{y}})\right] / d_{j} .
$$

In our experiments, we used the same heuristic as in Cez̧ik and L'Ecuyer to select the $d_{j}$ 's: We took $d_{j}=3$ when the SL corresponding to the considered cut was less than $0.5, d_{j}=2$ when it was between 0.5 and 0.65 , and $d_{j}=1$ when it was greater than 0.65 . When we need a subgradient for a period-specific empirical SL $\left(\hat{g}_{p}\right.$ or $\hat{g}_{k, p}$ ), the finite difference is formed only for those components of $\mathbf{y}$ corresponding to the given period; the other elements of $\overline{\mathbf{q}}$ are set to zero. This heuristic introduces inaccuracies, because $\hat{g}_{p}$ and $\hat{g}_{k, p}$ depend in general on the staffing of all periods up to $p$ or even $p+1$, but it reduces the work significantly.

Computing $\overline{\mathbf{q}}$ via (3) requires $I P+1$ simulations of $n$ days each. This is by far the most timeconsuming part of the algorithm. Even for medium-size problems, these simulations can easily require an excessive amount of time. For this reason, we use yet another important short-cut: We generally use a smaller value of $n$ for estimating the subgradients than for checking feasibility. (The latter requires a single $n$-day simulation experiment.) That is, we compute each $\hat{g}\left(\overline{\mathbf{y}}+d_{j} \mathbf{e}_{j}\right)$ in (3) using $n_{0}<n$ days of simulation, instead of $n$ days. In most of our experiments (including those reported in this paper), we have used $n_{0} \approx n / 10$.

With all these approximations and the simulation noise, we recognize that the vector $\overline{\mathbf{q}}$ thus obtained is only a heuristic guess for a subgradient. It may fail to be a subgradient. In that case the cut (2) may remove feasible staffing solutions including the optimal one, and this may lead our algorithm to a suboptimal schedule; Atlason et al. (2004) and Cez̧ik and L'Ecuyer give examples of this. For this reason, it is a good idea to run the algorithm more than once with different streams of random numbers and/or slightly different parameters, and retain the best solution found.

At each step of the algorithm, after adding new linear cuts, we solve a relaxation of $\left(\mathrm{SP}_{n}\right)$ in which the SL constraints have been replaced by a set of linear constraints. This is an integer programming (IP) problem. But when the number of integer variables is large, we just solve it as a linear program (LP) instead, because solving the IP becomes too slow. To recover an integer solution, we select a threshold $\delta$ between 0 and 1 ; then we round up (to the next integer) the real numbers whose fractional part is larger than $\delta$ and we truncate (round down) the other ones. These two versions of the CP algorithm are denoted CP-IP and CP-LP.

When we add new cuts, we give priority to the cuts associated with the global SL constraints, followed by aggregate ones specific to a call type, followed by aggregate ones specific to a period, 
followed by the remaining ones. This is motivated by the intuitive observation that the more aggregation we have, the smoother is the empirical SL function, because it involves a larger number of calls. So its gradient is less likely to oscillate and the vector $\mathbf{q}$ defined earlier is more likely to be a subgradient. Moreover, in the presence of abandonments, the SL functions tend to be non-concave in the areas where the SL is very small, and very small SL values tend to occur less often for the aggregated measures than for the more detailed ones that were averaged. Adding cuts that strengthen the aggregate SL often helps to increase the small SL values associated with specific periods and call types.

After adding enough linear cuts, we eventually end up with a feasible solution for $\left(\mathrm{SP} 0_{n}\right)$. This solution may be infeasible for (P0) (because of random noise, especially if $n$ is small) or may be feasible but suboptimal for (P0) (because one of the cuts may have removed the optimal solution of $(\mathrm{P} 0)$ from the feasible set of $\left.\left(\mathrm{SP}_{n}\right)\right)$. To try improving our solution to $\left(\mathrm{SP}_{n}\right)$, we perform a local search around it. In the CP-LP version, before launching this local search, the solution must be rounded to integers. This is done using a threshold $\delta$ as explained earlier. To determine this threshold, we perform a binary search over the interval $[0,1]$, up to an accuracy of 0.01 , to find the largest value of $\delta$ that yields a feasible integer solution for $\left(\mathrm{SP}_{n}\right)$.

The local search proceeds by iteratively considering longer simulations to check the feasibility of the solutions that it examines. The number of days used in these simulations, $n_{1}$, starts from a value $n_{2}$ (smaller than $n$ ) specified as an input parameter and increases at each iteration by $50 \%$ of this value. Each iteration of the local search tries to solve $\mathrm{SP} 0_{n_{1}}$, in three phases. In the first phase, the current solution is checked again for feasibility with the new value of $n_{1}$ and agents are added at minimum cost until feasibility has been restored, if required. In the second phase, we attempt to reduce the cost of the solution by removing one shift at a time (we try each combination of shift type and agent type), until none of the possibilities is feasible. We further attempt to reduce the cost in the third phase by iteratively considering switch moves in which we try to replace an agent/shift pair by another one with smaller cost; the candidates for the switch moves are drawn at random, at each step, and the phase terminates when a maximum number of consecutive moves without improvement is reached (we used 40 for that number). After the third phase, the current solution is tested for feasibility in a simulation of duration $n$ days. If it is feasible or if a time limit has been reached, the local search terminates, otherwise $n_{1}$ is increased and a new iteration is performed. Thus, at the end of the local search procedure, we have a feasible solution for either $\mathrm{SP} 0_{n_{1}}$ or $\mathrm{SP} 0_{n}$. The reason for using shorter, but increasingly long, simulations in the local search is the need to find some balance between limiting the time required to evaluate a large number of candidate solutions and ensuring the feasibility of the solutions considered (it is pointless to spend time examining a large number of solutions if they all turn up to be infeasible).

If we start the cutting plane algorithm with a full relaxation of $\left(\mathrm{SP}_{n}\right)$ (no constraint at all), the optimal solution of this relaxation is $\mathbf{y}=\mathbf{0}$. The functions $\hat{g}$ are not concave at $\mathbf{0}$, and we cannot 
get subgradients at that point, so we cannot start the algorithm from there. As a heuristic to quickly remove this area where the staffing is too small and the SL is non-concave, we restrict the set of admissible solutions a priori by imposing (extra) initial constraints. To do that, we impose that for each period $p$, the skill supply of the available agents covers at least $\alpha_{k}$ times the total load for each call type $k$ (defined as the arrival rate of that call type divided by its service rate), where each $\alpha_{k}$ is a constant, usually close to 1 . Finding the corresponding linear constraints is easily achieved by solving a max flow problem in a graph. See Cez̧ik and L'Ecuyer for the details. A pseudocode of the entire algorithm is provided in the on-line appendix.

\section{Computational Results}

In order to assess the performance of the proposed algorithm, as well as the impact of flexibility on solutions, a number of problem instances were solved with the proposed algorithm and the two-step (TS) method. These instances were constructed to be representative of real-life call centers, based on suggestions from people at Bell Canada. Their general setting is characterized as follows, unless stated otherwise.

The call center opens at 8:00 AM and closes at 5:00 PM; the working day is divided into $P=36$ 15-minute periods. Shifts vary in length between 6.5 hours ( 26 periods) and 9 hours (36 periods) and include a 30-minute lunch break near the middle and two 15-minute coffee breaks, one prelunch and one post-lunch. A shift is specified by five attributes: length, start time, time between the shift start and the beginning of the pre-lunch break (break 1 delay), lunch break start time, and time between the end of the lunch break and the beginning of the post-lunch break (break 3 delay). Table 1 shows the possible values of these attributes. There are 105 shifts of type 1, 45 shifts of type 6 , and 27 shifts for each of the five other types, for a total of 285 shifts.

Call arrivals are assumed to obey a stationary Poisson process over each period, for each call type, and independent across call types. The profile of the arrival rates in the different periods are inspired from observations in real-life call centers at Bell Canada (Avramidis et al. 2004). They are plotted separately for each instance. All service times are exponential with service rate $\mu=8$ calls per hour. Patience times have a mixture distribution: the patience is 0 with probability 0.001 , and with probability 0.999 , it is exponential with rate 0.1 per minute. The routing policy is an agents' preference-based router (Buist and L'Ecuyer 2005). These assumptions are not all very realistic; for example, the arrival streams of different call types are likely to be dependent, and the service times are usually non-exponential. But these simplifications should not affect much our algorithm.

For most instances, we only consider aggregate service level constraints for each period. These require that at least $80 \%$ of all the calls received during the period be answered within 20 seconds (i.e., we have $\tau_{p}=20$ seconds and $l_{p}=0.8$ for each $p$; these are typical values used in many call centers, often because there are SL regulations based on these values). The satisfaction of 


\begin{tabular}{|c|c|c|c|c|c|}
\hline Type & length & shift start & break 1 delay & lunch start & break 3 delay \\
\hline \hline & & $8: 00$ & $1: 30,1: 45,2: 00$ & $12: 00,12: 30$ & $1: 30,1: 45,2: 00$ \\
1 & $7: 30$ & $8: 00$ & $1: 30,1: 45,2: 00$ & $13: 00$ & $1: 30,1: 45$ \\
& & $8: 30,9: 00,9: 30$ & $1: 30,1: 45,2: 00$ & $12: 00,12: 30,13: 00$ & $1: 30,1: 45,2: 00$ \\
\hline 2 & $7: 45$ & $9: 15$ & $1: 30,1: 45,2: 00$ & $12: 00,12: 30,13: 00$ & $1: 30,1: 45,2: 00$ \\
\hline 3 & $8: 00$ & $9: 00$ & $1: 30,1: 45,2: 00$ & $12: 00,12: 30,13: 00$ & $1: 30,1: 45,2: 00$ \\
\hline 4 & $8: 15$ & $8: 45$ & $1: 30,1: 45,2: 00$ & $12: 00,12: 30,13: 00$ & $1: 30,1: 45,2: 00$ \\
\hline 5 & $8: 30$ & $8: 30$ & $1: 30,1: 45,2: 00$ & $12: 00,12: 30,13: 00$ & $2: 00,2: 15,2: 30$ \\
\hline 6 & $9: 00$ & $8: 00$ & $3: 00,3: 15,3: 30$ & $12: 00,13: 30,14: 00$ & $1: 15,1: 30,1: 45$ \\
& & & $3: 45,4: 00$ & & \\
\hline 7 & $6: 30$ & $10: 00$ & $1: 30,1: 45,2: 00$ & $13: 00,13: 30,14: 00$ & $0: 45,1: 00,1: 15$ \\
\hline
\end{tabular}

Table 1: Description of the 285 shifts for our examples

these constraints implies that the global constraint with $\tau=20$ seconds and $l=0.8$ is automatically satisfied, but we still require this explicitly, because this constraint plays a key role in the cuttingplane algorithm. In some cases, we also impose disaggregate SL constraints for each (call type, period) combination $(k, p)$ with $\tau_{k, p}=20$ seconds and $l_{k, p}=0.5$ for all $k$ and $p$. Note that this in turn implies the satisfaction of aggregate SL constraints for each call type $k$ with $\tau_{k}=20$ seconds and $l_{k}=0.5$. In practice, the aim of these disaggregated constraints is to avoid gross SL imbalance between the different call types or periods. Their target levels are typically lower than for the global constraint.

The formula used to compute agents' costs accounts for both the number of skills in the agent's skill set and the length of the shift being worked:

$$
c_{i q}=\left(1+\left(\eta_{i}-1\right) \varsigma\right) l_{q} / 30 \text { for all } i \text { and } q,
$$

where $l_{q}$ is the length (in periods) of shift $q, 30$ is the number of periods in a "standard" 7.5-hour shift, $\eta_{i}$ is the cardinality of $S_{i}$, and $\varsigma$ is an instance-specific parameter that represents the cost associated with each agent skill.

We first compare the two solution methods described (i.e., TS and CP) on three instances that correspond to a small (section 4.1), a medium-sized (section 4.2) and a larger call center (section 4.3). For the medium-size center, two variants are considered: $M_{1}$, in which only aggregate SL constraints considered, and $M_{2}$ with aggregate and disaggregate SL constraints. For the larger center, we also examine the impact of having a longer working day. This is motivated by the idea that the available shift types and the SL constraints may have a significant impact on the performance of the algorithm, as well as on the cost of the solution.

Both TS and CP use CPLEX 9.0 to solve the optimization problems. To allow a fair comparison of the methods, we allocate the same CPU time "budget" to each. Considering the nature of the algorithms, this cannot be done by simply stopping them when this time limit is reached. Instead, we must carefully adjust, by trial and error, the number $n$ of simulated days, which is a key parameter 
of both methods, to obtain running times close to the target budget. It is clear that one would not use such a procedure in a practical context, but this is necessary for the comparative study. For each instance, we consider several different budgets, since we expect that a higher value of $n$ will produce more accurate and more stable results. Furthermore, in each case, $r$ replications of each method/budget combination are performed to account for the random elements in both methods.

In the first phase of $\mathrm{TS}$, to simulate each individual period of the call center and evaluate the results of the simulations, we use the batch means method (Law and Kelton 2000). Each batch is constituted by a minimum number of 30 simulation time units and statistical observations are collected on a minimum of 50 batches, using 2 warmup batches before starting to collect statistics.

Final solutions obtained by the two methods were simulated for $n_{*}=50,000$ days as an additional (much more stringent) feasibility test, and each solution was declared feasible or not according to the result of this test, i.e., according to the feasibility of $\left(\mathrm{SP}_{n_{*}}\right)$.

For each instance (or variant), results are summarized in a table with the following column headings: budget, the given CPU time budget; $n$, the number of simulated days for checking feasibility when adding cutting planes and for the local search at the end of the algorithm (we took $n_{0} \approx n / 10$ ); $n_{2}$, the starting number of days in local search simulations; $C P U_{a v g}$, the average CPU time per replication; Min cost and Med cost, which are respectively the minimum and median costs of all feasible solutions for $\left(\mathrm{SP}_{n_{*}}\right)$ obtained by this method over the $r$ replications; $P^{*}$, the percentage of replications that returned a feasible solution for $\left(\mathrm{SP}_{n_{*}}\right)$; and $P_{1}^{*}$, the percentage that returned a feasible solution with cost within $1 \%$ of the best known feasible solution (the lowest-cost feasible solution for $\left(\mathrm{SP}_{n_{*}}\right)$ generated by either algorithm, over all replications and CPU time budgets, in all experiments that we have done, including those described in Section 4.4 and several others). We also report the maximum relative violation gap (in percent) observed in a SL constraint for each type of constraints; $G_{\text {period }}$ and $G_{\text {call,period }}$ refer respectively to violations of SL constraints for periods and for individual (call type, period) combinations.

\subsection{A small call center}

This instance has $K=2$ call types and $I=2$ agent types, with $S_{1}=\{1\}$ and $S_{2}=\{1,2\}$. Agent costs are computed by setting the parameter $\varsigma$ equals to 0.2 in formula 4 . Arrival rates for the two call types are plotted in Figure 1. All SL constraints are enforced in this instance. Four different CPU time budgets were considered: 3, 15, 30 and 60 minutes. Results, based on $r=32$ replications, are displayed in Table 2.

Several observations can be made from Table 2. First, CP-IP is able to find cheaper feasible solutions more often than TS for all CPU budgets, except for very short CPU time. As expected, the probability of finding good solutions with CP-IP improves as the CPU budget and length of simulation are increased. On the other hand, it is interesting to observe that the performance of TS does not really improve with a larger time budget. This points out that finding a better staffing 


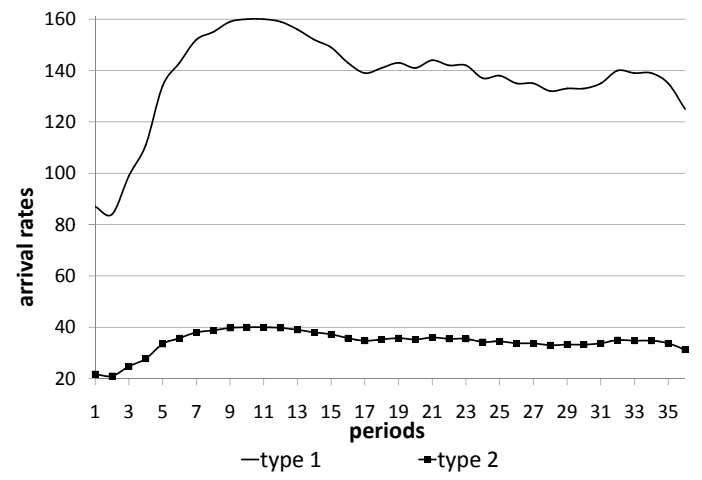

Figure 1: Small center: arrival rates

\begin{tabular}{|c|cccc|cc|cc|cc|}
\hline $\begin{array}{c}\text { Budget } \\
(\mathrm{min})\end{array}$ & Algorithm & $n$ & $n_{2}$ & $\begin{array}{c}\mathrm{CPU}_{\text {avg }} \\
\text { sec. }\end{array}$ & $\begin{array}{c}\text { Min } \\
\text { cost }\end{array}$ & $\begin{array}{c}\text { Med } \\
\text { cost }\end{array}$ & $P_{1}^{*}$ & $P^{*}$ & $G_{\text {period }}$ & $G_{\text {call,period }}$ \\
\hline \hline 3 & CP-IP & 120 & 50 & 191 & 36.47 & 37.67 & 0 & 34 & 2.65 & 3.22 \\
& TS & 600 & & 205 & 35.57 & 35.57 & 0 & 3 & 1.46 & 0 \\
\hline 15 & CP-IP & 1500 & 800 & 916 & 35.13 & 36.10 & 9 & 66 & 0.36 & 0.47 \\
& TS & 2800 & & 831 & 35.59 & 35.64 & 0 & 12 & 0.73 & 0 \\
\hline 30 & CP-IP & 2000 & 1000 & 1751 & 35.19 & 35.99 & 3 & 69 & 0.36 & 0.07 \\
& TS & 5500 & & 1739 & 35.59 & 35.67 & 0 & 9 & 0.56 & 0 \\
\hline 60 & CP-IP & 3000 & 1500 & 3031 & 35.17 & 35.77 & 22 & 78 & 0.42 & 0 \\
& TS & 9000 & & 2810 & 35.51 & 35.66 & 0 & 12 & 0.58 & 0 \\
\hline
\end{tabular}

Table 2: Small center: results obtained with CP-IP and TS for different CPU time budgets

solution per period does not necessarily lead to a better scheduling solution. TS also has great difficulty finding feasible solutions, although constraint violations were almost always inferior to $1 \%$ (for both TS and CP-IP). In practice, a manager might be willing to use almost-feasible solutions, considering the fact that the center will always experience stochastic variation in the arrival process and the SL in any case. For this reason, it is probably useful to report slightly infeasible solutions in general, and not only the feasible ones.

Table 3 gives an aggregate view of the best feasible scheduling solutions obtained by CP-IP and TS in these runs. In this table, shift types correspond to the length of the shifts as indicated in Table 1. Both methods return solutions with the same number of agents (31), most of which are specialists (type 1). Further analysis of these solutions reveals that CP-IP schedules agents to slightly shorter shifts and uses more specialists than TS (23 vs 22), and thus gives a cheaper solution. We have also solved a variant of this problem in which both types of specialists were allowed. Under the same cost structure as before, the optimal solution for that case also uses 31 agents, but is somewhat cheaper (the optimal value is 34.45) since 26 agents are now specialists (again, 23 of these are type-1 specialists, while the three others handle type-2 calls). 


\begin{tabular}{|c|c|c|c|c|c|c|c|c|}
\hline Algorithm & Agent type & \multicolumn{7}{|c|}{ Shift type } \\
\cline { 3 - 8 } & & 1 & 2 & 3 & 4 & 5 & 6 & 7 \\
\hline CP-IP & 1 & 7 & 1 & 3 & 2 & 1 & 6 & 3 \\
& 2 & 2 & 1 & 0 & 0 & 1 & 4 & 0 \\
\hline TS & 1 & 7 & 1 & 2 & 2 & 1 & 7 & 2 \\
& 2 & 1 & 1 & 1 & 0 & 1 & 4 & 1 \\
\hline
\end{tabular}

Table 3: Small center: scheduling solutions

Service levels for the CP-IP solution from Table 3 are plotted in Figure 2. We see (1) a wide variation of the SL throughout the day and (2) that calls of type 1 have much better SL than those of type 2 . This imbalance can be explained by the fact that the type 1 calls can be answered by less expensive specialists, while type 2 calls must be handled by generalists. This observation highlights the fact that to ensure a fair treatment of all call types in a real-life setting, it is often necessary to include call-type specific SL constraints (either over the whole day or for each period) in the problem formulation.

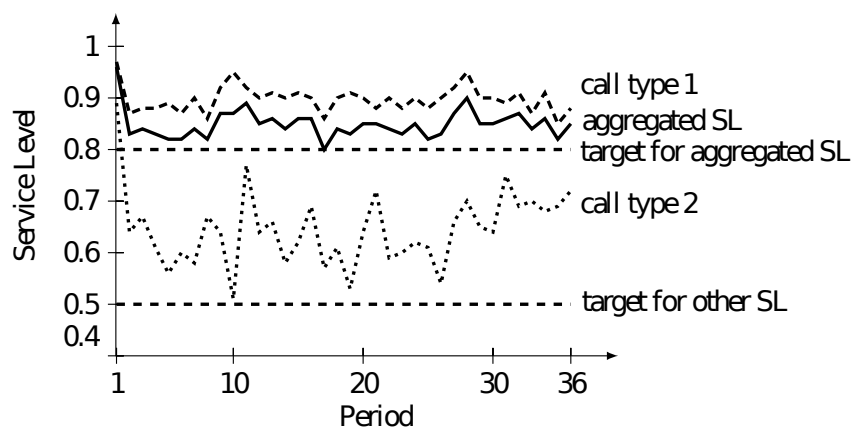

Figure 2: Small center: service levels by period

\subsection{A medium-sized call center}

In the medium-sized instances, there are $K=5$ call types and $I=15$ agent types. Five of the agents types are specialists handling a single call type, while the other ten are generalists handling between 2 and 5 call types. Details on skill sets can be found in Table 4 . The parameter $\varsigma$ used to compute agent costs in formula 4 is now equal to 0.1. Arrival rates for all call types are plotted in Figure 3.

As mentioned earlier, we consider two variants of this example: in $M_{1}$, only global and perperiod SL constraints are enforced, while $M_{2}$ also includes disaggregate SL constraints. Since in practice one may find it hard to satisfy all SL constraints and since real-life call center managers are often interested primarily in global SL, it seemed interesting to compare these two variants. For each of them, we performed $r=8$ replications for several CPU time budgets of 15, 30 and 60 minutes. Because solving the IP instances to optimality would require unacceptable running time, 


\begin{tabular}{|c|c|}
\hline Skill & Agent types \\
\hline 1 & $1,6,9,10,11,14,15$ \\
\hline 2 & $2,7,9,10,12,13,14,15$ \\
\hline 3 & $3,8,13,15$ \\
\hline 4 & $4,11,12,13,14,15$ \\
\hline 5 & $5,6,7,8,10,11,12,14,15$ \\
\hline
\end{tabular}

Table 4: Medium-sized center: skill sets

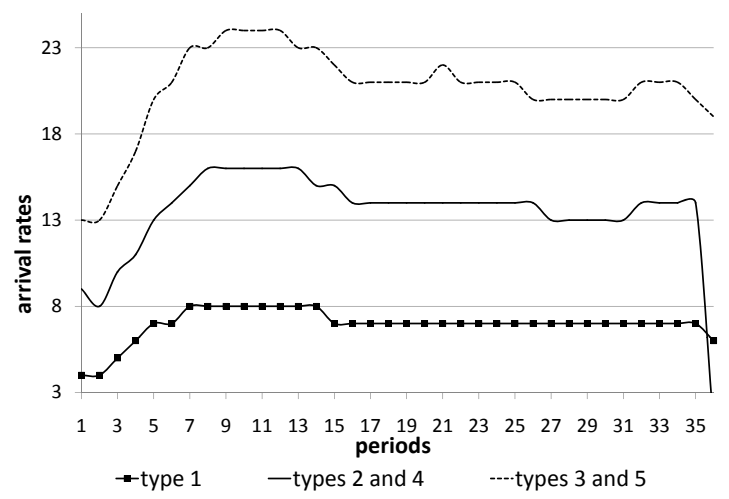

Figure 3: Medium-sized center: arrival rates

we experimented with two solution strategies: in the first, we used CP-LP instead of CP-IP, while in the second, we set a time limit varying from 10 to 90 seconds (for long budget) for the CPLEX solver in CP-IP. CP-IP generally requires fewer iterations than CP-LP, which compensates for the additional IP solving time. The results for $M_{1}$ and $M_{2}$ are summarized in Tables 5 and 6. Overall, the performance of CP-IP and CP-LP is similar: both methods have a good chance to find lower cost (5\% cheaper) feasible solutions than TS, but all the solutions obtained by TS are feasible. CP-IP and CP-LP had to execute more iterations to optimize $M_{2}$ and the solutions are a little more expensive than in $M_{1}$; this was expected since $M_{1}$ is a relaxation of $M_{2}$. An interesting conclusion that one could draw here is that the increase in cost incurred when imposing the disaggregate SL constraints is rather marginal. Again, it is worth noting that the performance of TS appears invariant to the CPU time, but also to the additional disaggregate SL constraints.

\subsection{A larger call center}

The larger center instances have $K=20$ call types and $I=35$ agent types. Twenty of the agents types are specialists handling a single call type, while the fifteen other types are generalists handling between 4 and 9 call types. Details on skill sets can be found in Avramidis et al. (2007b) and in the appendix. Agent costs are computed with $\varsigma=0.1$. We only consider SL constraints by period, plus the global SL constraint. Two CPU time budgets are examined: 5 and 10 hours. Because the size of 


\begin{tabular}{|c|cccc|cc|cc|c|}
\hline $\begin{array}{c}\text { Budget } \\
(\mathrm{min})\end{array}$ & Algorithm & $n$ & $n_{2}$ & $\begin{array}{c}\mathrm{CPU}_{\mathrm{avg}} \\
\text { sec. }\end{array}$ & $\begin{array}{c}\text { Min } \\
\text { cost }\end{array}$ & $\begin{array}{c}\text { Med } \\
\text { cost }\end{array}$ & $P_{1}^{*}$ & $P^{*}$ & $G_{\text {period }}$ \\
\hline \hline \multirow{3}{*}{15} & CP-IP & 200 & 80 & 764 & 20.54 & 20.99 & 0 & 62 & 0.85 \\
& CP-LP & 200 & 80 & 972 & 20.29 & 20.96 & 0 & 50 & 2.33 \\
& TS & 1000 & & 864 & 21.47 & 21.60 & 0 & 100 & 0 \\
\hline \multirow{3}{*}{30} & CP-IP & 600 & 100 & 1776 & 20.18 & 20.51 & 12 & 75 & 0.88 \\
& CP-LP & 600 & 100 & 1947 & 20.36 & 20.88 & 0 & 62 & 0.74 \\
& TS & 1333 & & 1494 & 21.43 & 21.61 & 0 & 100 & 0 \\
\hline \multirow{3}{*}{60} & CP-IP & 1000 & 400 & 2981 & 20.45 & 20.90 & 0 & 62 & 0.46 \\
& CP-LP & 1000 & 400 & 2987 & 20.51 & 21.26 & 0 & 75 & 0.25 \\
& TS & 4000 & & 3603 & 21.49 & 21.57 & 0 & 100 & 0 \\
\hline
\end{tabular}

Table 5: $M_{1}$ : results obtained with CP-LP and TS for different CPU time budgets

\begin{tabular}{|c|cccc|cc|cc|cc|}
\hline $\begin{array}{c}\text { Budget } \\
(\mathrm{min})\end{array}$ & Algorithm & $n$ & $n_{2}$ & $\begin{array}{c}\mathrm{CPU}_{\text {avg }} \\
\text { sec. }\end{array}$ & $\begin{array}{c}\text { Min } \\
\text { cost }\end{array}$ & $\begin{array}{c}\text { Med } \\
\text { cost }\end{array}$ & $P_{1}^{*}$ & $P^{*}$ & $G_{\text {period }}$ & $G_{\text {call,period }}$ \\
\hline \hline \multirow{3}{*}{15} & CP-IP & 200 & 80 & 664 & 20.80 & 21.53 & 0 & 37 & 0.43 & 0.21 \\
& CP-LP & 200 & 80 & 1146 & 20.57 & 21.81 & 0 & 62 & 0.98 & 2.28 \\
& TS & 1000 & & 903 & 21.47 & 21.59 & 0 & 100 & 0 & 0 \\
\hline \multirow{3}{*}{30} & CP-IP & 300 & 80 & 2188 & 20.92 & 21.89 & 0 & 62 & 0.32 & 0 \\
& CP-LP & 300 & 80 & 1827 & 20.65 & 21.22 & 0 & 62 & 1.02 & 0 \\
& TS & 1333 & & 1804 & 21.47 & 21.62 & 0 & 100 & 0 & 0 \\
\hline \multirow{3}{*}{60} & CP-IP & 400 & 100 & 3106 & 20.59 & 21.26 & 0 & 62 & 0.32 & 3.37 \\
& CP-LP & 400 & 100 & 4597 & 20.71 & 21.47 & 0 & 50 & 0.17 & 2.21 \\
& TS & 4000 & & 3604 & 21.53 & 21.59 & 0 & 100 & 0 & 0 \\
\hline
\end{tabular}

Table 6: $M_{2}$ : results obtained with CP-LP and TS varying the CPU time budget

the problem is too large to run CP-IP efficiently (even with limited IP solving time), we only execute CP-LP and perform $r=8$ replications.

One of our objectives with this example is to show that the performance of CP-LP does not depend much on the particular structure of the shifts. We thus consider again two variants: $L_{36}$, which uses the 9-hour working day and the same shift structure as the previous examples, and $L_{52}$, which has a working day starting at 8:00 AM and ending at 9:00 PM; in that variant, the total number of periods is 52 and all the shifts have a fixed length of 7.5 hours, thus yielding a total of 123 different shifts (considering also shifts starting at 1:00 PM and 1:30 PM in order to cover the additional periods). Arrival rates for the $L_{36}$ variant and the 36 first periods of $L_{52}$, follow exactly the same pattern as in the medium-size example (see Figure 3), with different scalings for the different call types; for $L_{52}$ they then decrease slowly during the last 16 periods. The results for $L_{36}$ are displayed in Table 7.

Each run of CP-LP has to execute in total around 20,000 simulations. Although the CPU budgets are several hours, each simulation is actually quite short (averages of 0.7 and $1.3 \mathrm{~second} / \mathrm{simulation}$ respectively). CP-LP has difficulty finding feasible solutions, even though constraint violations are 


\begin{tabular}{|c|cccc|cc|cc|c|}
\hline $\begin{array}{c}\text { Budget } \\
\text { (hours) }\end{array}$ & Algorithm & $n$ & $n_{2}$ & $\begin{array}{c}\mathrm{CPU}_{\text {avg }} \\
\text { min. }\end{array}$ & $\begin{array}{c}\text { Min } \\
\text { cost }\end{array}$ & $\begin{array}{c}\text { Med } \\
\text { cost }\end{array}$ & $P_{1}^{*}$ & $P^{*}$ & $G_{\text {period }}$ \\
\hline \hline 5 & CP-LP & 400 & 50 & 261 & 79.17 & 80.05 & 0 & 50 & 0.77 \\
& TS & 4000 & & 268 & 95.99 & 100.46 & 0 & 100 & 0 \\
\hline 10 & CP-LP & 500 & 80 & 506 & 78.38 & 79.44 & 12 & 38 & 0.65 \\
& TS & 6000 & & 602 & 96.64 & 100.30 & 0 & 100 & 0 \\
\hline
\end{tabular}

Table 7: $L_{36}$ : results obtained with CP-LP and TS for different CPU time budgets

\begin{tabular}{|c|cccc|cc|cc|c|}
\hline $\begin{array}{c}\text { Budget } \\
\text { (hours) }\end{array}$ & Algorithm & $n$ & $n_{2}$ & $\begin{array}{c}\mathrm{CPU}_{\text {avg }} \\
\text { min. }\end{array}$ & $\begin{array}{c}\text { Min } \\
\text { cost }\end{array}$ & $\begin{array}{c}\text { Med } \\
\text { cost }\end{array}$ & $P_{1}^{*}$ & $P^{*}$ & $G_{\text {period }}$ \\
\hline \hline 5 & CP-LP & 200 & 50 & 300 & 133.00 & 133.40 & 25 & 37 & 1.31 \\
& TS & 3000 & & 257 & 161.50 & 167.85 & 0 & 100 & 0 \\
\hline 10 & CP-LP & 400 & 50 & 696 & 133.70 & 134.15 & 12 & 25 & 0.48 \\
& TS & 4400 & & 561 & 158.60 & 163.40 & 0 & 100 & 0 \\
\hline
\end{tabular}

Table 8: $L_{52}$ : results obtained with CP-LP and TS for different CPU time budgets

typically very small. TS always finds feasible solutions, but the solutions returned are on average $25 \%$ more expensive than those obtained with CP-LP. Surprisingly, increasing the CPU budget does not improve the performance of TS. This confirms our observations of the previous section regarding the limited performance of TS. Optimizing periods independently does not seem to lead to a better scheduling solution. On closer examination of the best scheduling solutions obtained by the two methods, we find that the CP-LP solution is less expensive because it covers the demand with only 52 agents compared to 62 for TS.

The results for the $L_{52}$ variant are reported in Table 8 . On this larger problem, each run of CP-LP has to execute in total around 35,000 simulations. Because each simulation needs to be short (less than 1 second), there is a higher probability of ending up with an infeasible solution. However, the violation gap decreases as the time budget increases. All the solutions returned by TS were declared feasible, but they are $20 \%$ more expensive. When we examine the best solutions found by CP-LP and by TS for the $L_{52}$ instance, we first remark that CP-LP uses only 94 agents compared to 104 for TS. We also note that 6 agents in the CP-LP solution are specialists, while there is 1 in the TS solution. Furthermore, TS uses 32 expensive generalists with 7 skills or more compared to only 22 for CP-LP. These three factors combined explain the large difference in cost.

Our motivation for investigating the 52-period example was to verify that CP-LP performed correctly for instances with a different shift structure. Our results confirm this, but at the same time they highlight one of the potential shortcomings of the approach, which is that, because of simulation noise, when there is a large number of constraints, one often ends up with no feasible solution, even though several near-feasible solutions may have been identified. We address this issue next. 


\subsection{Getting more feasible results}

Empirical results show that, as problem instances become larger and more complex, there is a definite possibility that $\mathrm{CP}$ would return a set of low-cost, but only nearly-feasible solutions. While this may be acceptable in some practical settings, it is nonetheless annoying to be unable to provide the call center manager with a solution that meets all his/her requirements. A simple and attractive way of tackling this problem consists in slightly increasing the right-hand side value of the SL constraints when applying the algorithm (except obviously for the final long simulation that is used to determine the feasibility of solutions). It should be noted that this idea is not specific to the CP procedure and could therefore be applied with any other solution approach.

We first tested this idea on the $L_{52}$ instance, using values of 0.81 as target SL for all periods. We combined these tests with experiments on the value of the threshold $\delta$ used for rounding continuous solutions to integer ones in CP-LP. The rationale for investigating different values of $\delta$ is that the rounding procedure introduces a heuristic element in what would otherwise be an exact procedure and that selecting the best value for this threshold is far from obvious.

In our experiments, we considered three different values of $\delta(0.5,0.6$ and 0.7$)$ for CPU budgets of 5 and 10 hours, and we ran 8 replications in each case. The chance of obtaining good feasible solutions has greatly improved and even had all 8 solutions feasible for one test case. The solutions also tend to cost slightly more. The results are summarized in Table 9 . These results show that the value selected for $\delta$ seems to have a small impact on the quality of the solutions obtained. Using a $\delta$ closer to 1 results in a lower incumbent rounded solution in CP-LP and may not represent well the incumbent LP solution, in particular when there are few agents per (group,shift)-combination. This can lead to bad cuts in early stages of CP-LP, which happened in the test case $\delta=0.7$. In fact, it seems to be much more important to make sure that the runs that are made do produce feasible solutions, in order to have a larger set to choose from.

\begin{tabular}{|c|c|cc|c|}
\hline$\delta$ & $\begin{array}{c}\text { CPU budget } \\
\text { hours }\end{array}$ & $\begin{array}{c}\text { Min } \\
\text { cost }\end{array}$ & $\begin{array}{c}\text { Med } \\
\text { cost }\end{array}$ & $P^{*}$ \\
\hline \hline 0.5 & 5 & 133.90 & 135.35 & 50 \\
& 10 & 133.70 & 134.85 & 100 \\
\hline 0.6 & 5 & 133.70 & 135.00 & 87 \\
& 10 & 134.50 & 135.15 & 100 \\
\hline 0.7 & 5 & 139.00 & 140.20 & 87 \\
& 10 & 136.70 & 139.15 & 75 \\
\hline
\end{tabular}

Table 9: $L_{52}$ : results obtained with a target SL of 0.81

We then ran CP-LP with the original 0.80 target value for different values of $\delta$. These tests clearly showed that modifying $\delta$ alone was not sufficient to consistently obtain feasible solutions, since more than half of these runs returned infeasible solutions. We also ran the algorithm with a 
target SL value of 0.81 for the other instances (keeping the value of $\delta$ unchanged at 0.5 ). The results can be summarized as follows:

- For the small center, all runs returned feasible solutions (except for very short time budget). The solutions tend to cost slightly more than the ones obtained previously, but a better solution was found with a cost of 35.11 .

- For the medium-sized instances, almost every run produced a feasible solution, but we did not find a better solution.

- For the $L_{36}$ instance, all runs yielded a feasible solution, and we found a better solution with a cost of 78.27 .

Overall, slightly increasing the value of the SL target value is a useful device for making sure that the method will return feasible solutions. There is no guarantee that this will provide a better solution, but it may very well do so, especially when there is a large number of constraints. The variability of our results highlights once again the stochastic nature of the algorithm, which cannot be avoided considering the significant amount of noise in the simulations.

\subsection{The impact of flexibility}

We performed another series of numerical experiments to quantify empirically the impact of the flexibility provided by a rich set of shift types. Those experiments were performed on the small center of subsection 4.1. We considered three sets of shift types: the original one with all 285 shifts, a slightly reduced one with 267 shifts, obtained by deleting the 26-period and some 36-period shifts, and adding some 35-period ones, and finally a much more restricted set in which we only allow the 105 7.5-hour shifts. The staffing solutions corresponding to the best scheduling solutions obtained for these three cases are plotted in Figure 4, along with the optimal staffing solution computed by considering each period individually. Three main conclusions can be drawn from this figure:

1. As shown by the solution with 285 shifts, if enough flexibility is introduced in the set of available shifts, it is possible to find schedules that track closely the staffing requirements.

2. Even a slight decrease in flexibility (e.g., by going from 285 to 267 shifts) can lead to a significant overstaffing in some periods.

3. Schedules with a relatively small number of fixed-length shifts (the 105-shift case) are bound to suffer from major overstaffing.

It follows that, while the complexity of the scheduling problem significantly increases with the number of available shifts, there are definite benefits to be reaped from the introduction of more varied shift types. 


\section{Conclusion}

We have proposed in this paper a simulation-based methodology to optimize agent scheduling over one day in a multiskill call center. Even though the use of common random numbers reduces the simulation noise (or variance) significantly, there is still a fair amount of randomness in the solution provided by the algorithm, mainly due to the fact that the simulation length must be kept short (because the estimation of each subgradient requires simulations at up to thousands of different parameter values). Yet, to our knowledge, better solutions are found with this approach than with any other method we know. In particular, during the development of the cutting plane algorithm, we also implemented simultaneously a metaheuristic method based on neighborhood search combined with queueing approximation, along the lines of Avramidis et al. (2006), but we were unable to make it competitive for solving the scheduling problem.

In practice, one may run the algorithm a few times (e.g., overnight) to obtain a few solutions and retain the best found. We also showed that by slightly perturbing the SL targets, it is possible to overcome some of the problems caused by the presence of the simulation noise and thus to greatly increase the probability of obtaining feasible, high-quality solutions.

Future research on this problem includes the search for faster ways of estimating the subgradients, refining the algorithm to further reduce the noise in the returned solution, and extending the technique to simultaneously optimize the scheduling and the routing of calls (via dynamic rules).

\section{Acknowledgements}

This research has been supported by Grants OGP-0110050, OGP38816-05, and CRDPJ-320308 from NSERC-Canada, and a grant from Bell Canada via the Bell University Laboratories, to the third and fourth authors, and a Canada Research Chair to the fourth author. The second author benefited from a scholarship provided jointly by NSERC-Canada, the Fonds québécois de la recherche sur

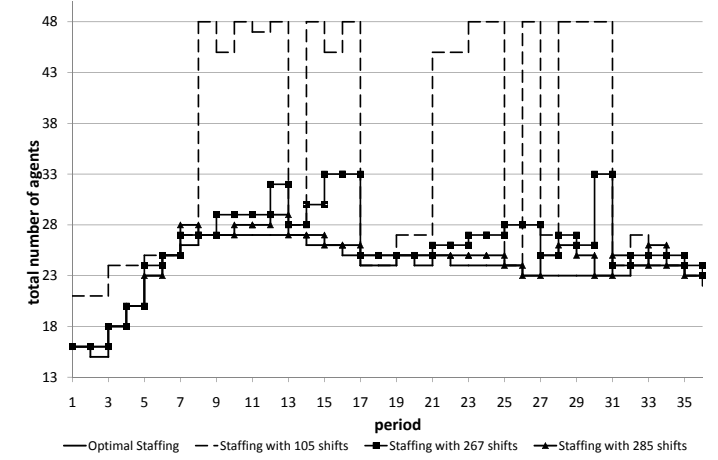

Figure 4: Small center: staffing solutions with 105, 267 and 285 shift types 
la nature et les technologies, and Bell Canada. The fifth author benefited from the support of the University of Calabria and the Department of Electronics, Informatics and Systems (DEIS), and her thanks also go to professors Pasquale Legato and Roberto Musmanno.

\section{References}

J. Atlason, M. A. Epelman, and S. G. Henderson. Call center staffing with simulation and cutting plane methods. Annals of Operations Research, 127:333-358, 2004.

A. N. Avramidis, A. Deslauriers, and P. L'Ecuyer. Modeling daily arrivals to a telephone call center. Management Science, 50(7):896-908, 2004.

A. N. Avramidis, W. Chan, and P. L'Ecuyer. Staffing multi-skill call centers via search methods and a performance approximation. Submitted, Revised in May 2007, 2006.

A. N. Avramidis, M. Gendreau, P. L'Ecuyer, and O. Pisacane. Simulation-based optimization of agent scheduling in multiskill call centers. In Proceedings of the 2007 Industrial Simulation Conference. Eurosis, 2007a.

A. N. Avramidis, M. Gendreau, P. L'Ecuyer, and O. Pisacane. Optimizing daily agent scheduling in a multiskill call center. Technical report, Publication CIRRELT-2007-44, CIRRELT, Université de Montréal, Montreal, 2007b.

S. Bhulai, G. Koole, and A. Pot. Simple methods for shift scheduling in multi-skill call centers, 2007. Manuscript, available at http://www.cs.vu.nl/ koole/research.

L. Brown, N. Gans, A. Mandelbaum, A. Sakov, H. Shen, S. Zeltyn, and L. Zhao. Statistical analysis of a telephone call center: A queueing-science perspective. Journal of the American Statistical Association, 100:36-50, 2005.

E. Buist and P. L'Ecuyer. A Java library for simulating contact centers. In Proceedings of the 2005 Winter Simulation Conference, pages 556-565. IEEE Press, 2005.

U. D. o. L. Bureau of Labor Statistics. Occupational Outlook Handbook, Customer Service Representatives, 2006-07 Edition. 2007. Available online at http://www.bls.gov/oco/ocos 280. htm (last accessed February 14, 2007).

M. T. Cez̧ik and P. L'Ecuyer. Staffing multiskill call centers via linear programming and simulation. Management Science, 54:310-323. 
CRTC. Final standards for quality of service indicators for use in telephone company regulation and other related matters, 2000. Canadian Radio-Television and Telecommunications Commission, Decision CRTC 2000-24. See http://www.crtc.gc.ca/archive/ENG/Decisions/ 2000/DT2000-24.htm.

N. Gans, G. Koole, and A. Mandelbaum. Telephone call centers: Tutorial, review, and research prospects. Manufacturing and Service Operations Management, 5:79-141, 2003.

L. V. Green, P. J. Colesar, and J. Soares. An improved heuristic for staffing telephone call centers with limited operating hours. Production and Operations Management, 12:46-61, 2003.

S. Halfin and W. Whitt. Heavy-traffic limits for queues with many exponential servers. Operations Research, 29:567-588, 1981.

S. Henderson and A. Mason. Rostering by iterating integer programming and simulation. In Proceedings of the 1998 Winter Simulation Conference, volume 1, pages 677-683, 1998.

A. Ingolfsson, E. Cabral, and X. Wu. Combining integer programming and the randomization method to schedule employees. Technical report, School of Business, University of Alberta, Edmonton, Alberta, Canada, 2003. Preprint.

O. B. Jennings, A. Mandelbaum, W. A. Massey, and W. Whitt. Server staffing to meet time-varying demand. Management Science, 42(10):1383-1394, 1996.

E. G. Keith. Operator scheduling. AIIE Transactions, 11(1):37-41, 1979.

G. Koole and A. Mandelbaum. Queueing models of call centers: An introduction. Annals of Operations Research, 113:41-59, 2002.

A. M. Law and W. D. Kelton. Simulation Modeling and Analysis. McGraw-Hill, New York, third edition, 2000.

P. L'Ecuyer. SSJ: A Java Library for Stochastic Simulation, 2004. Software user's guide, Available at http://www.iro.umontreal.ca/ lecuyer.

P. L'Ecuyer, R. Simard, E. J. Chen, and W. D. Kelton. An object-oriented random-number package with many long streams and substreams. Operations Research, 50(6):1073-1075, 2002.

V. Mehrotra. Ringing up big business. ORMS Today, 24(4):18-24, October 1997.

A. Pot, S. Bhulai, and G. Koole. A simple staffing method for multi-skill call centers, 2007. Manuscript, available at http://www.cs.vu.nl/ koole/research. 
R. Stolletz and S. Helber. Performance analysis of an inbound call center with skill-based routing. OR Spectrum, 26:331-352, 2004.

G. M. Thompson. Labor staffing and scheduling models for controlling service levels. Naval Research Logistics, 8:719-740, 1997.

S. Vogel. A stochastic approach to stability in stochastic programming. Journal of Computational and Applied Mathematics, 56:65-96, 1994. 